\title{
A Mathematical Model for Spread of COVID-19 in the World
}

\author{
Pirooz Mohazzabi, Gabrielle Richardson, Gwendolyn Richardson \\ Department of Mathematics and Physics, University of Wisconsin-Parkside, Kenosha, WI, USA \\ Email:mohazzab@uwp.edu
}

How to cite this paper: Mohazzabi, P., Richardson, G. and Richardson, G. (2021) A Mathematical Model for Spread of COVID-19 in the World. Journal of Applied Mathematics and Physics, 9, 1890-1895.

https://doi.org/10.4236/jamp.2021.98122

Received: June 2, 2021

Accepted: August 8, 2021

Published: August 11, 2021

Copyright $\odot 2021$ by author(s) and Scientific Research Publishing Inc. This work is licensed under the Creative Commons Attribution International License (CC BY 4.0).

http://creativecommons.org/licenses/by/4.0/

\begin{abstract}
In this article, the validity of a previously developed model for Coronavirus pandemic is verified by testing it against the global infection cases complied by the World Health Organization. The results further support the validity of the model and its assumptions as was previously verified by the United States data.
\end{abstract}

\section{Keywords}

Coronavirus, Pandemic, World, COVID-19

\section{Introduction}

Epidemic diseases have haunted mankind forever and have wiped out large fractions of the populations throughout the history. The Spanish flu during 1918-1919 infected nearly one-third of the world population and claimed at least 50 million lives [1]. In more recent years, the longest lasting pandemic of HIV/AIDS, which peaked during the period of 2005-2012, has claimed more than 36 million lives worldwide [2] [3].

The currently active COVID-19 pandemic, which outbroke in December of 2019 [4], has so far infected 172 million people worldwide and has claimed 3.69 million lives [5]. Due to its economic and political impacts, COVID-19 pandemic has prompted a number of investigations into the dynamics of its spread and predictions of its future development. For example, Leung et al. [6] studied the transmissibility and severity of the first wave of the disease in China. Kissler et al. [7] projected the transmission dynamics of the disease through the postpandemic period. Haushofer and Metcalf [8] investigated the effectiveness of various interventions in a pandemic. Sanche et al. [9] studied the high contagiousness and rapid spread of the coronavirus. Finally, Kaxiras and Neofotistos 
[10] investigated the multiple epidemic wave model of the COVID-19 pandemic.

Recently, we developed a new model for the Coronavirus pandemic. This model contains only two adjustable parameters as compared to as many as 5 parameters in other models [11]. The model was tested against the number of Coronavirus cases in the United States during the period of January 2020 to January 2021, and the results showed good agreement between the model and the data. In this work, we extend the application of the model to the number of cases in the entire world, for which enough data was not available during the previous work.

\section{The Model}

Recently, we developed a simple model for the spread of Coronavirus pandemic (COVID-19). This model is based on the normal uninhibited population growth,

$$
\frac{\mathrm{d} N}{\mathrm{~d} t}=k N
$$

where $N$ is the instantaneous population and $k$ is the growth constant, which is the probability of a member of the population to double per unit time. In a pandemic, however, the rate of disease spread at any time is proportional to the number of the remaining healthy population,

$$
\frac{\mathrm{d} n}{\mathrm{~d} t}=k(N-n)
$$

where $N$ is the total population, which is a constant, and $n$ is the infected population at time $t$. Furthermore, in this model, instead of treating $k$ as a constant, it is assumed to be proportional to the fraction of the infected population [11]. Thus,

$$
k=k_{0} \frac{n}{N}
$$

where now $k_{0}$ is a constant. Therefore, the differential equation for the growth of the infected population becomes

$$
\frac{\mathrm{d} n}{\mathrm{~d} t}=k(N-n)=k_{0} \frac{n}{N}(N-n)
$$

which reduces to

$$
\frac{\mathrm{d} n}{n(N-n)}=\frac{k_{0}}{N} \mathrm{~d} t
$$

Integration of this equation gives

$$
\frac{n}{N-n}=A \mathrm{e}^{k_{0} t}
$$

where $A$ is related to the integration constant. Applying the initial conditions $n(0)=n_{0}$, gives

$$
A=\frac{n_{0}}{N-n_{0}}
$$


Therefore, Equation (6) reduces to [11]

$$
n(t)=\frac{n_{0} N}{n_{0}+\left(N-n_{0}\right) \mathrm{e}^{-k_{0} t}}
$$

Furthermore, since in a pandemic situation, the initial infected population is much smaller than the total population, $n_{0} \ll N$, this equation further reduces to

$$
n(t)=\frac{n_{0} N}{n_{0}+N \mathrm{e}^{-k_{0} t}}
$$

which gives the number of the infected population as a function of time. The S-shape graph of this equation is usually referred to as the logistic growth curve [11] [12].

\section{Application of the Model to World COVID-19 Data}

As stated in a previous section, we recently tested the model described above against the COVID-19 data in the United States during the period of January 2020 to January 2021. The results showed good agreement between the model and the data even though the model contains only two adjustable parameters, namely $n_{0}$ and $N$.

At the time that the previous work was completed, we could not find reliable comprehensive data for the entire world. However, recently we were able to find enough worldwide data to test this model against them [13]. To do so, we complied the world data in increments of 7-days due to this period correlating to the common measurement of time known as a "week" making the data more comprehensible. Thus, in Table 1, Day 1 corresponds to December 30, 2019 and Day 533 corresponds to June 20, 2021.

Using a non-linear least-squares analysis, we fitted Equation (9) to the data in Table 1 via the adjustable parameters $n_{0}$ and $k$ as described in reference [11]. For the total population $N$ we used the current population of the world, $7.7 \times 10^{9}$ (7.7 billion). This resulted in the following values of the parameters:

$$
n_{0}=(7.01 \pm 0.63) \times 10^{6} \text { and } k=(6.37 \pm 0.19) \times 10^{-3} \text { day }^{-1}
$$

Figure 1 shows the graph of Equation (9) with these parameters together with the actual world data from Table 1.

\section{Conclusions}

As can be seen from Figure 1, the overall agreement between our model and the World Coronavirus data is reasonably good. We do see, however, some variations in the data resulting in the model overestimating in the actual data in some regions and underestimating in some other regions. This is attributed to changes in the dynamics of the disease spread due to modifications in the parameters of the pandemic such as mask mandates, social distancing, and vaccination efforts. The willingness of population to adhere to parameter modifications as well as the revisions made by the $\mathrm{CDC}$ and $\mathrm{WHO}$ regarding mandates also influenced 


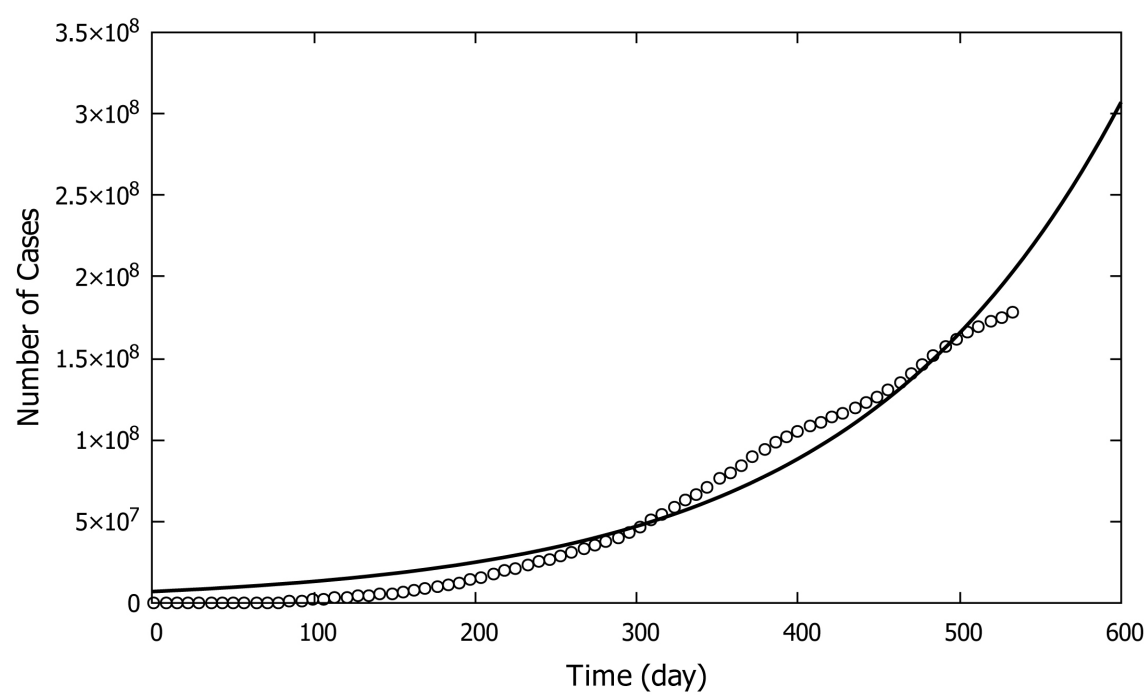

Figure 1. Worldwide COVID cases as a function of time.

Table 1. Number of the world coronavirus cases from December 30, 2019 to June 20, 2021 [13]. The data obtained from WHO is continuously revised as more data come in meaning the data which was collected originally may be slightly altered if viewed at a further date.

\begin{tabular}{|c|c|c|c|c|c|c|c|}
\hline Day & Cases & Day & Cases & Day & Cases & Day & Cases \\
\hline 1 & 1 & 141 & $5,225,157$ & 281 & $37,710,539$ & 421 & $113,561,976$ \\
\hline 8 & 45 & 148 & $5,947,720$ & 288 & $40,268,140$ & 428 & $116,312,221$ \\
\hline 15 & 137 & 155 & $6,786,661$ & 295 & $43,290,917$ & 435 & $119,279,244$ \\
\hline 22 & 2061 & 162 & $7,675,625$ & 302 & $46,759,504$ & 442 & $122,600,095$ \\
\hline 29 & 14,644 & 169 & $8,694,929$ & 309 & $50,556,433$ & 449 & $126,432,603$ \\
\hline 36 & 37,657 & 176 & $9,833,792$ & 316 & $54,636,216$ & 456 & $130,535,153$ \\
\hline 43 & 69,375 & 183 & $11,137,846$ & 323 & $58,781,647$ & 463 & $135,122,255$ \\
\hline 50 & 79,005 & 190 & $12,566,581$ & 330 & $62,849,434$ & 470 & $140,398,755$ \\
\hline 57 & 87,426 & 197 & $14,133,450$ & 337 & $67,027,757$ & 477 & $146,142,306$ \\
\hline 64 & 107,466 & 204 & $15,877,378$ & 344 & $71,394,221$ & 484 & $151,882,470$ \\
\hline 71 & 161,243 & 211 & $17,724,078$ & 351 & $76,044,276$ & 491 & $157,375,571$ \\
\hline 78 & 314,646 & 218 & $19,568,304$ & 358 & $80,182,403$ & 498 & $162,207,358$ \\
\hline 85 & 675,304 & 225 & $21,459,711$ & 365 & $84,325,552$ & 505 & $166,083,578$ \\
\hline 92 & $1,174,742$ & 232 & $23,272,553$ & 372 & $89,371,212$ & 512 & $169,647,532$ \\
\hline 99 & $1,729,560$ & 239 & $25,128,039$ & 379 & $94,230,121$ & 519 & $172,678,347$ \\
\hline 106 & $2,264,263$ & 246 & $27,091,286$ & 386 & $98,498,696$ & 526 & $175,346,072$ \\
\hline 113 & $2,819,049$ & 253 & $29,028,553$ & 393 & $102,292,594$ & 533 & $177,875,936$ \\
\hline 120 & $3,367,817$ & 260 & $31,121,103$ & 400 & $105,520,024$ & & \\
\hline 127 & $3,953,175$ & 267 & $33,223,301$ & 407 & $108,392,203$ & & \\
\hline 134 & $4,551,838$ & 274 & $35,342,832$ & 414 & $110,878,947$ & & \\
\hline
\end{tabular}


fluctuations in data. These fluctuations, however, average out resulting in the effective parameters $n_{0}$ and $N$ given by Equation (10).

The agreement between Equation (9) and the world data on COVID-19 cases is indicative of validity of the model and the corresponding underlying assumptions, which is further evidenced by agreement of the model with the United States data alone. Furthermore, since the model does not depend on the specifics of COVID-19, it can be applied to any pandemic in general.

\section{Acknowledgements}

This work was supported in part by a URAP grant from the University of Wisconsin-Parkside.

\section{Conflicts of Interest}

The authors declare no conflicts of interest regarding the publication of this paper.

\section{References}

[1] CDC Influenza (Flu). https://www.cdc.gov/flu/pandemic-resources/1918-pandemic-h1n1.html

[2] MP Honline. https://www.mphonline.org/worst-pandemics-in-history/

[3] Centers for Disease Control and Prevention. https://www.cdc.gov/flu/pandemic-resources/basics/past-pandemics.html

[4] Archived: WHO Timeline, COVID-19. https://www.who.int/news/item/27-04-2020-who-timeline---covid-19

[5] Statistics. https://www.google.com/search?q=covid+infections+worldwide+total\&rlz=1C1CH BF enUS936US936\&oq=COVID+infections + worl\&aqs=chrome.2.0j69i57j017j0i390 .12323j0j15\&sourceid $=$ chrome\&ie $=$ UTF- 8

[6] Leung, K., Wu, J., Liu, D. and Leung, G. (2020) First-Wave COVID-19 Transmissibility and Severity in China outside Hubei after Control Measures, and Second-Wave Scenario Planning: A Modelling Impact Assessment, Lancet, 395, 1382-1393. https://doi.org/10.1016/S0140-6736(20)30746-7

[7] Kissler, S.M., Tedijanto, C., Goldstein, E., Grad, Y.H. and Lipsitch, M. (2020) Projecting the Transmission Dynamics of SARS-CoV-2 through the Postpandemic Period. Science, 368, 860-868. https://doi.org/10.1126/science.abb5793

[8] Haushofer, J. and Metcalf, C.J.E. (2020) Which Interventions Work Best in a Pandemic? Science, 368, 1063-1065. https://doi.org/10.1126/science.abb6144

[9] Sanche, S., Lin, Y.T., Xu, C., Romero-Severson, E., Hengartner, N. and Ke, R. (2020) High Contagiousness and Rapid Spread of Severe Acute Respiratory Syndrome Coronavirus 2. Emerging Infectious Diseases, 26, 1470-1477. https://doi.org/10.3201/eid2607.200282

[10] Kaxiras, E. and Neofotistos, G. (2020) Multiple Epidemic Wave Model of the COVID-19 Pandemic: Modeling Study. Journal of Medical Internet Research, 22, e20912. https://doi.org/10.2196/20912

[11] Mohazzabi, P., Richardson, G. and Richardson, G. (2021) A Model for Coronavirus Pandemic. Journal of Infectious Diseases and Epidemiology, 7, 197. 
https://doi.org/10.23937/2474-3658/1510197

[12] Dym, C.L. and Ivey, E.S. (1980) Principles of Mathematical Modeling. Academic Press, New York, 178.

[13] WHO Coronavirus (COVID-19) Dashboard. https://covid19.who.int/ 